\title{
Factors associated with aspiration pneumonia and preventive measures in hospitalized elderly: a systematic review of observational studies
}

\author{
Daniela de Arimateia Rosa Santos ${ }^{1}$ \\ https://orcid.org/0000-0003-2121-1328 \\ Débora de Paula Silva Arcanjo ${ }^{1}$ \\ https://orcid.org/0000-0002-0886-8379 \\ Geiza Maria Bezerra de Melo e Silva1 \\ https://orcid.org/0000-0002-2222-0675 \\ Amanda Louize Félix Mendes ${ }^{2}$ \\ https://orcid.org/0000-0002-2079-4499 \\ Simonize Cunha Barreto de Mendonça ${ }^{3}$ \\ https://orcid.org/0000-0003-2055-8901 \\ Thales Rafael Correia de Melo Lima ${ }^{4}$ \\ https://orcid.org/0000-0003-0009-4206 \\ Carlos Kazuo Taguchi ${ }^{5}$ \\ https://orcid.org/0000-0003-2428-0134 \\ Brenda Carla Lima Araújo 5
}

Hospital Universitário de Sergipe, Programa Multiprofissional da Saúde do Adulto e do Idoso, Aracaju, Sergipe, Brasil.

Maternidade Nossa Senhora de Lourdes, Aracaju, Sergipe, Brasil.

Hospital Universitário de Sergipe da Universidade Federal de Sergipe, Aracaju, Sergipe, Brasil.

Universidade Federal de Sergipe, Programa de Pós-graduação Profissional em Gestão e Inovação Tecnológica em Saúde, Aracaju, Sergipe, Brasil.

Universidade Federal de Sergipe Departamento de Fonoaudiologia, São Cristóvão, Sergipe, Brasil.

Institution: Hospital Universitário de Sergipe, Aracaju, Sergipe, Brazil.

Conflict of interests: Nonexistent

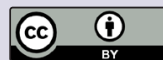

Received on: March 13, 2020 Accepted on: September 08, 2020

Corresponding address: Daniela de Arimateia Rosa Santos.

Rua A, Loteamento Mangueira, 277 Bairro: Dezoito do Forte

CEP: 49072-629 - Aracaju, Sergipe, Brasil

E-mail: fgadanielarimateia@gmail.com

\section{ABSTRACT}

Purpose: to identify associated factors and preventive measures for aspiration pneumonia in hospitalized elderly patients.

Methods: the PPOT (population, predictor, outcome, and type of study) was the strategy used to define the eligibility criteria: (1) Population: people over 60 years of age; (2) predictor: hospitalized patients (3) Outcomes: associated factors and measures to prevent aspiration pneumonia; (4) Type of study: Observational Studies (transversal and case-control). We performed the data collection in the PubMed, Lilacs, Scielo, Google Scholar, Science Direct, and Web of Science databases. Observational studies on factors associated with aspiration pneumonia and preventive measures in the elderly, without language restriction and available in full, were included. Data on authors/year/country, objective, methodology, sample, and results, were collected. The risk of bias was assessed, according to the JBI Critical Appraisal Checklist.

Results: a case-control and two cross-sectional studies were analyzed. One paper presented a smaller sample with 86 patients, and another presented a larger sample comprising 443 patients. The median age was 77 years old.

Conclusion: factors such as severe stroke, dysphagia, advanced age, male gender, coronary heart disease, lower Glasgow coma scale score, use of mechanical ventilation for more than 48 hours, and aspiration of colonized gastric juice were associated with aspiration pneumonia.

Keywords: Speech, Language and Hearing Sciences; Aged; Aspiration Pneumonia; Deglutition Disorders; Systematic Review 


\section{INTRODUCTION}

The increasing life expectancy is changing the world age scenario. In Brazil, it is estimated that there will be some 32 million elderly people in 2025 and it is estimated that, in 2050, this group will represent $22 \%$ of the Brazilian population and $16 \%$ of people worldwide. The natural process of aging brings with it great functional, psychic, and cognitive limitations that interfere in the health conditions of the elderly, causing hospitalizations, which can lead to or result in infectious conditions, as in cases of aspiration pneumonia ${ }^{1,2}$.

Aspiration pneumonia, one of the most commonly diagnosed infections in the geriatric population, occurs when the patient aspirates colonized secretions present in the oropharyngeal cavity and/or colonized gastric residues in the lower respiratory tract. Its incidence of morbidity and mortality is $15 \%$ and $18 \%$, respectively, which can increase the hospitalization time of a patient at an average of 7 to 9 days. It is one of the main factors of rising hospitalization costs worldwide ${ }^{2-5}$.

A recent study investigated hospital costs in patients with pneumonia admitted to the Intensive Care Unit (ICU). Of the patients hospitalized in this unit, $71 \%$ were diagnosed with aspiration pneumonia, the third largest cause of rising hospital costs, together with antibiotic therapy ${ }^{6}$.

The prevalence of aspiration risk in hospitals is high, around $56 \%^{2}$. Infectious surveillance is one of the patient's safety measures, which is understood as the reduction, to an acceptable minimum, of the risks associated with health care ${ }^{7}$. The application of preventive protocols regarding the risk of aspiration, together with an early evaluation of the speech therapist, is cost-effective with savings reaching almost
US\$ 169.83 per hospitalization - an essential measure for patient safety ${ }^{3}$.

Considering the incidence of aspiration pneumonia, increased hospitalization time, and a higher cost with antibiotic therapy, it is essential to care for hospitalized elderly patients and it is necessary to create aspiration risk prevention programs, especially with the participation of speech therapists. This professional can aid in the damage prevention to health and the rehabilitation of these patients, contributing to a safer assistance ${ }^{6-9}$. Thus, in the present study, a systematic review to identify associated factors and preventive measures for aspiration pneumonia in hospitalized elderly patients, was performed. The hypothesis of the study was that dysphagia and prolonged intubation were a risk factor for aspiration pneumonia in hospitalized elderly.

\section{METHODS}

The present study focused on answering the following question: What factors and preventive measures were adopted for aspiration pneumonia in hospitalized elderly?

We used the following PPOT (population, predictor, outcome, and type of study) elements to define the eligibility criteria: (1) Population: people over 60 years of age, regardless of age and gender; (2) Predictor: the hospitalized patients; (3) Outcomes: risk and/ or measures to prevent aspiration pneumonia, liquid substances, secretions, and gastroesophageal reflux; (4) Type of study: Observational Studies, including cross-sectional and case-control (Table 1), available in full and published in the last 10 years, without language restriction.

Table 1. Description of the search strategy

\begin{tabular}{ccc}
\hline Search Strategy & Definition & Description \\
\hline P & Population & People over 60 years of age, regardless of age and gender. \\
P & Predictor & People who were hospitalized. \\
0 & Outcomes & Risk and/or measures to prevent aspiration pneumonia, liquid substances, secretion and \\
T & Study type & Observational Studies, including cross-sectional, case control and accuracy studies. \\
\hline
\end{tabular}


We performed a systematic search to identify relevant studies published in PubMed, Latin American and Caribbean Literature in Health Science (LILACS), Scientific Electronic Library Online (SCIELO), ScienceDirect, and Web of Science. A grey-literature search was conducted using Google Scholar and the first 100 results were analyzed. The reference lists of all eligible studies and reviews were thoroughly evaluated to identify additional studies for inclusion.
The structured search strategy was adapted according to the search database, with the purpose to cover the largest number of possible studies in the literature and used the following terms: "Aspiration Pneumonia" OR "Pneumonia, Aspiration" OR "Aspiration Pneumonia" OR "Pneumonia, Aspiration" AND "Factor, Risk" OR "Factors, Risk" OR "Risk Factor" OR "Risk Factors" AND "prophylaxis" OR "preventive therapy" OR "control" OR "prevention and control" (Table 2). The search occurred between November 3 to December 25, 2018.

Table 2. Research strategies in electronic databases

\begin{tabular}{|c|c|c|}
\hline Databases & Search strategy (November 2018) & No. \\
\hline PubMed & 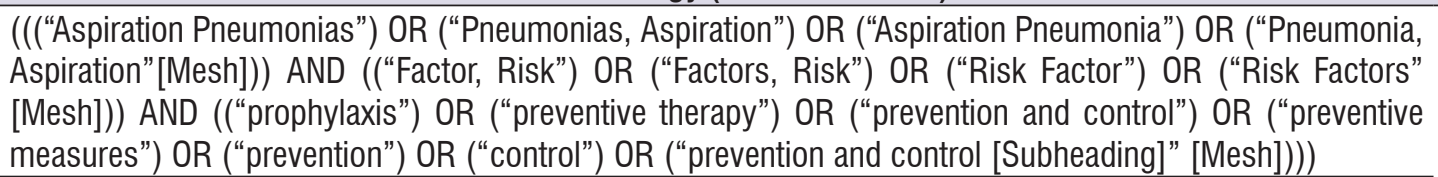 & 418 \\
\hline Web OF & $\begin{array}{l}\text { (((Aspiration Pneumonias) OR (Pneumonias, Aspiration) OR (Aspiration Pneumonia) OR (Pneumonia, } \\
\text { Aspiration)) AND ((Factor, Risk) OR (Factors, Risk) OR (Risk Factor) OR (Risk Factors)) AND ((prophylaxis) }\end{array}$ & \\
\hline Science & $\begin{array}{l}\text { OR (preventive therapy) OR (prevention and control) OR (preventive measures) OR (prevention) OR (control) } \\
\text { OR (prevention and control))) }\end{array}$ & 368 \\
\hline Science Direct & $\begin{array}{l}\text { ((“"Aspiration Pneumonias”) OR (“Pneumonias, Aspiration”) OR ("Aspiration Pneumonia”) OR (“Pneumonia, } \\
\text { Aspiration”) AND ((“Factor, Risk”) OR (“Factors, Risk”) OR (“Risk Factor”) OR (“Risk Factors”)) AND } \\
\text { (("prophylaxis") OR ("preventive therapy”) OR ("prevention and control”) OR ("preventive measures”) OR } \\
\text { (“prevention”) OR (“control”) OR ("prevention and control”))) }\end{array}$ & 436 \\
\hline LiLacs & $\begin{array}{l}\text { (((Aspiration Pneumonias) OR (Pneumonias, Aspiration) OR (Aspiration Pneumonia) OR (Pneumonia, } \\
\text { Aspiration)) AND ((Factor, Risk) OR (Factors, Risk) OR (Risk Factor) OR (Risk Factors)) AND ((prophylaxis) } \\
\text { OR (preventive therapy) OR (prevention and control) OR (preventive measures) OR (prevention) OR (control) } \\
\text { OR (prevention and control))) }\end{array}$ & 12 \\
\hline Scielo & $\begin{array}{l}\text { (((Aspiration Pneumonias) OR (Pneumonias, Aspiration) OR (Aspiration Pneumonia) OR (Pneumonia, } \\
\text { Aspiration)) AND ((Factor, Risk) OR (Factors, Risk) OR (Risk Factor) OR (Risk Factors)) AND ((prophylaxis) } \\
\text { OR (preventive therapy) OR (prevention and control) OR (preventive measures) OR (prevention) OR (control) } \\
\text { OR (prevention and control))) }\end{array}$ & 03 \\
\hline Google Scholar & $\begin{array}{l}\text { "Aspiration Pneumonias" OR "Pneumonias, Aspiration" OR "Aspiration Pneumonia" OR "Pneumonia, } \\
\text { Aspiration"AND "Factor, Risk" OR "Factors, Risk" OR "Risk Factor" OR "Risk Factors" AND "prophylaxis" OR } \\
\text { "preventive therapy" OR "prevention and control" OR "preventive measures" OR "prevention” OR "control" } \\
\text { OR "prevention and control " }\end{array}$ & 100 \\
\hline Summation $\Sigma$ & & 1337 \\
\hline
\end{tabular}

\section{Selection Criteria}

This study followed the guidelines of the Preferred Reporting Items for Systematic Reviews (PRISMA) ${ }^{10}$. The selection of the studies had two stages. Initially, the titles and abstracts were analyzed independently by two reviewers, who were not blind to authors or journals. At this moment, the studies that did not meet the eligibility criteria were excluded.

The second phase consisted of examining the full texts of the preliminarily eligible studies to verify whether they met the pre-established criteria. Discarded articles were recorded separately, explicitly stating the reasons for their exclusion.

To expand the number of eligible papers recovered, no filters were used in the search. Two review authors independently examined the results of the research and identified potentially relevant studies based on the title and the abstract. The relevant studies were read in full and selected according to the eligibility criteria. Disagreements between the two reviewers were dealt with by consensus or by a third reviewer. 
Studies that were not related to the topic, studies with adults and/or children, studies that did not present the sample age variation, case series studies, clinical trials, monographs, dissertations, thesis, literature and systematic reviews, books, and book chapters, were excluded.

\section{Data Analysis}

Using a standard form, each reviewer extracted the data containing accurate study information: authors/year/country, objective, methodology, study design, sample, and results. When necessary, a thirdparty professional was consulted to assess possible disagreements.

The risk of bias analysis of the selected studies was performed using the tool recommended by JBI Critical Appraisal Checklist ${ }^{11}$ specific for cross-sectional and case-control studies.

To evaluate the studies, the following items were used: Cross-Sectional Studies: 1) Were the criteria for inclusion in the sample clearly defined? 2) Were the study subjects and the setting described in detail? 3) Was the exposure measured in a valid and reliable way? 4) Were objective, standard criteria used for measurement of the condition? 5) Were confounding factors identified? 6) Were strategies to deal with confounding factors stated? 7) Were the outcomes measured in a valid and reliable way? 8) Was appropriate statistical analysis used? Case-control studies: 1) Were the groups comparable other than the presence of disease in cases or the absence of disease in controls? 2) Were cases and controls matched appropriately? 3) Were the same criteria used for identification of cases and controls? 4) Was exposure measured in a standard, valid, and reliable way? 5) Was exposure measured in the same way for cases and controls? 6) Were confounding factors identified? 7) Were strategies to deal with confounding factors stated? 8) Were outcomes assessed in a standard, valid, and reliable way for cases and controls? 9) Was the exposure period of interest long enough to be meaningful? 10) Was appropriate statistical analysis used? The risk of bias was classified as 'Low' when the results reached $70 \%$ or more of the "yes" score, 'Moderate' when it varied from $50 \%$ to $69 \%$ of "yes", and 'High' when the results presented up to $49 \%$ of the "yes" score.

\section{LITERATURE REVIEW}

The first phase of the studies selection resulted in 1,337 records distributed among the electronic databases. After reading the titles and abstracts, 1,180 studies were excluded because they did not meet the eligibility criteria. Thus, 157 articles were selected for the analysis of the full text. After this stage, 154 studies were excluded for the following reasons: 1- the study population ( $\mathrm{n}=59$ studies); 2 - being thesis and reviews ( $n=25$ studies); 3 - they are clinical trials and experimental studies ( $n=10$ studies); 4 - they are studies with more than 10 years of publication $(n=60$ studies).

Finally, three articles ${ }^{12-14}$ met the eligibility criteria and were included in this systematic review. The hands-on search in study references did not identify additional articles.

For the qualitative synthesis of the systematic review, two cross-sectional studies ${ }^{13,14}$ and a casecontrol study ${ }^{12}$ published in the years $2012^{12}, 2013^{13}$, and $2015^{14}$ were analyzed, with one article presenting a smaller sample with $86^{14}$ subjects and one presenting a larger sample with 443 patients $^{13}$. The mean age of study participants was 77 years old, with a minimum age of 60 and a maximum of 96 years old. Only two articles reported the hospitalization period, ranging from 4 to 812 days and 1-109 days, with a mean of 25.7 \pm 7.2 days $^{12}$. The papers are summarized in Table 3.

The results of the qualitative analysis of the three articles are described in Table 4. Each item was classified as yes, no, unclear, or not applicable. Two studies presented a low risk of bias and one presented a moderate risk of bias. The item marked "Unclear" indicates whether the exposure was measured in a standard, valid and reliable way ${ }^{12}$.

Studies have also reported that, besides the severity of stroke and dysphagia, other factors are related to the development of aspiration pneumonia, such as advanced age, male gender, coronary disease, and lower Glasgow Coma Scale (GCS) score ${ }^{12,13}$. Mechanical ventilation for more than 48 hours and aspiration of gastric juice were also present, with the latter related to the development of late aspiration pneumonia, defined as the aspiration pneumonia developed after more than 5 days of mechanical ventilation. These results indicate the presence of colonizing microorganisms in the gastrointestinal tract, with microaspiration being a risk factor for the development of aspiration pneumonia ${ }^{14}$. 


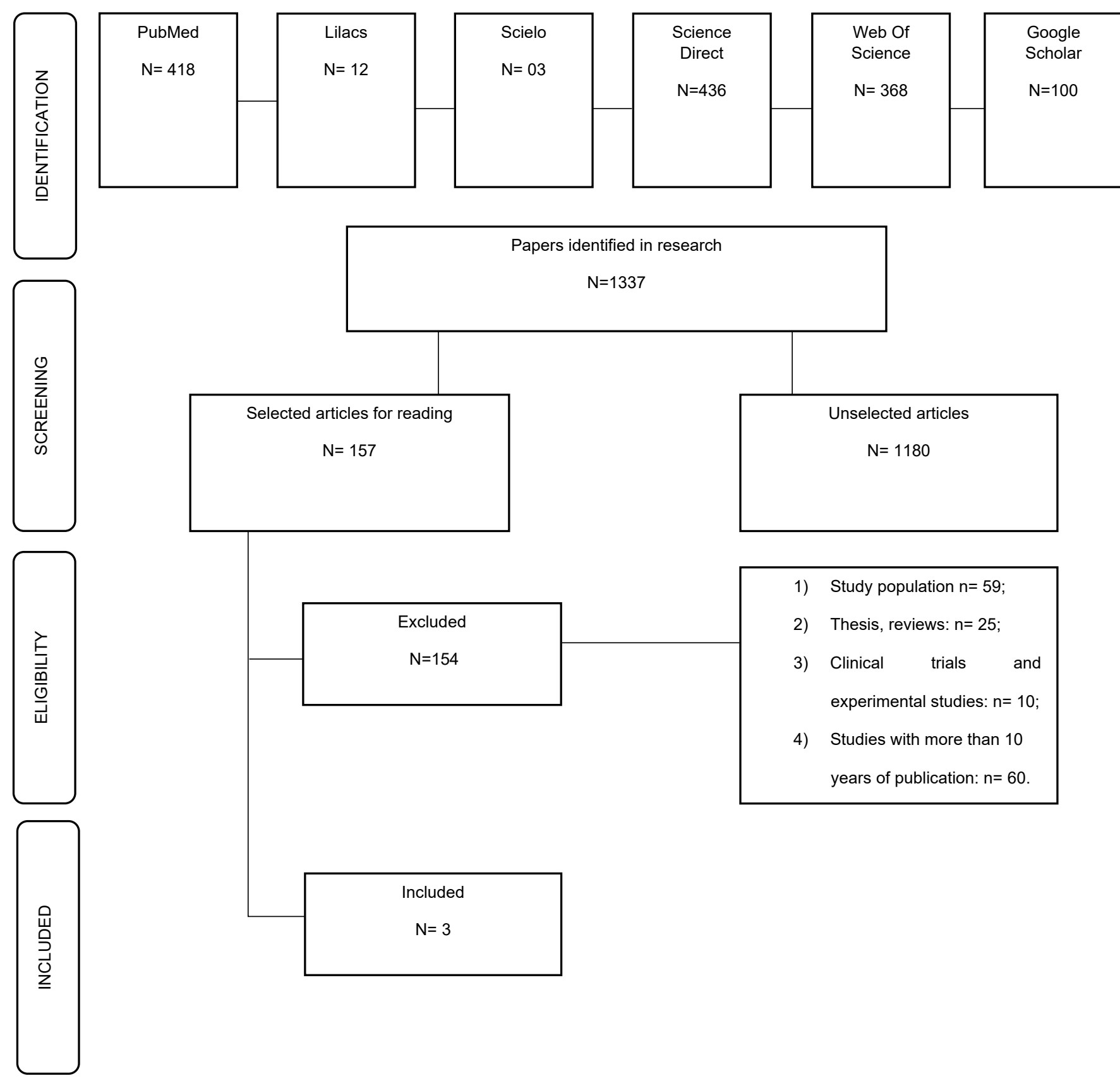

Figure 1. Flowchart of paper selection process, according to PRISMA 
Table 3. Summary of main features and results of eligible studies ${ }^{12-14}$

\begin{tabular}{|c|c|c|c|c|c|c|}
\hline $\begin{array}{l}\text { Authors } \\
\text { / Year / } \\
\text { Country }\end{array}$ & Objective & Metodology & $\begin{array}{l}\text { Type of } \\
\text { study }\end{array}$ & $\begin{array}{c}\text { Sample } \\
\text { (n) }\end{array}$ & Age & Results \\
\hline $\begin{array}{l}\text { Ishigami } \\
\text { et al. }^{.2} \\
2012 \\
\text { Japan }\end{array}$ & $\begin{array}{l}\text { To assess the association of } \\
\text { elevated blood pressure in the } \\
\text { acute phase with the incidence of } \\
\text { pneumonia. }\end{array}$ & $\begin{array}{l}\text { Three groups of patients were } \\
\text { compared: } 10 \text { elderly patients } \\
\text { with acute ischemic stroke with } \\
\text { severe hypertension, } 43 \text { patients } \\
\text { with moderate hypertension } \\
\text { and } 65 \text { normotensive or slightly } \\
\text { hypertensive controls. Data were } \\
\text { collected on known risk factors, } \\
\text { type of IVCA and underlying chronic } \\
\text { conditions. }\end{array}$ & $\begin{array}{l}\text { Case- } \\
\text { Control }\end{array}$ & $\begin{array}{l}58 \hat{\jmath} \\
\text { and } \\
60+\end{array}$ & $\begin{array}{l}72-96 \\
\text { years }\end{array}$ & $\begin{array}{l}\text { AP in patients with acute } \\
\text { ischemic stroke was } \\
\text { higher in the moderately } \\
\text { hypertensive group, } \\
\text { along with lower GCS } \\
\text { score, dysphagia, male } \\
\text { gender and coronary } \\
\text { heart disease. }\end{array}$ \\
\hline $\begin{array}{c}\text { Masrur } \\
\text { et al. }^{13} \\
2013 \\
\text { United } \\
\text { States }\end{array}$ & $\begin{array}{l}\text { To examine: the incidence and } \\
\text { results of dysphagia screening } \\
\text { along with the factors associated } \\
\text { with its occurrence; the incidence } \\
\text { and results in patients with APand } \\
\text { the risk factors associated with } \\
\text { their occurrence; the relationship } \\
\text { between DSand APin patients with } \\
\text { IVCA. }\end{array}$ & $\begin{array}{l}\text { Consecutive hospitalizations for } \\
\text { stroke were identified through } \\
\text { retrospective identification using } \\
\text { the International Classification of } \\
\text { Diseases and the Ninth Revision of } \\
\text { discharge codes, surveillance of } \\
\text { emergency records, ward records } \\
\text { or neurological consultations. }\end{array}$ & $\begin{array}{l}\text { cross- } \\
\text { sectional }\end{array}$ & $\begin{array}{l}212 \AA \\
\text { and } \\
231+\end{array}$ & $\begin{array}{l}61-82 \\
\text { years }\end{array}$ & $\begin{array}{l}\text { Of the eligible patients, } \\
5.7 \%(17,906) \\
\text { developed AP. When } \\
\text { compared to patients } \\
\text { without AP, patients with } \\
\text { BP were older, had an } \\
\text { increase in hospital stay } \\
\text { and mortality. }\end{array}$ \\
\hline $\begin{array}{l}\text { Liu et al. }{ }^{14} \\
2015 \\
\text { China }\end{array}$ & $\begin{array}{l}\text { To verify the gastropulmonary route } \\
\text { of infection in the pathogenesis } \\
\text { of PA in ventilated patients, and } \\
\text { to develop a diagnostic tool for } \\
\text { microaspiration; Investigate the } \\
\text { prevalence of microaspiration in } \\
\text { order to develop effective prevention } \\
\text { strategies. }\end{array}$ & $\begin{array}{l}\text { Were used field gel electrophoresis } \\
\text { with macrorestrictionby pulse } \\
\text { to verify the infection pathway } \\
\text { and to investigate the prevalence } \\
\text { of microaspiration in ventilated } \\
\text { elderly patients expected to receive } \\
\text { mechanical ventilation over } 48 \\
\text { h, enrolled from October } 2009 \text { to } \\
\text { January } 2012 \text {. }\end{array}$ & $\begin{array}{l}\text { cross- } \\
\text { sectional }\end{array}$ & $\begin{array}{l}53 \hat{\jmath} \\
\text { and } \\
33 ㅇ\end{array}$ & $\begin{array}{l}60-92 \\
\text { years }\end{array}$ & $\begin{array}{l}\text { Almost } 30 \% \text { of the } \\
\text { MVAP were caused by } \\
\text { microaspiration. About } \\
58.3 \% \text { of patients with } \\
\text { gastric colonization } \\
\text { developed VAP. }\end{array}$ \\
\hline
\end{tabular}

T: Male gender; ㅇ: Female gender; AP: aspiration pneumonia; STP: Speech Therapist; FR: Forms Recognition Test; DS: Dysphagia Screening; IVCA: ischemic stroke;GCS: Glasgow Coma Scale; MVAP: aspiration pneumonia associated with mechanical ventilation after 5 days; PAV: aspiration pneumonia associated with mechanical ventilation

No studies on the prevention of aspiration pneumonia in hospitalized elderly were found. We found the following risk factors: stroke, dysphagia, male gender, advanced age, coronary diseases, lower GCS score ${ }^{12,13}$, use of mechanical ventilation for more than 48 hours, and aspiration of colonized gastric juice ${ }^{14}$.

Regarding the factors associated with pneumonia, studies corroborate with the findings of this research. Severe stroke and lower GCS score ${ }^{12,13}$ were cited together with the Scale of the National Institute of Health Stroke (NIHSS) score, which signals the severity of the stroke numerically ${ }^{12}$. Other studies have found as pneumonia independent predictors the ischemic stroke, advanced age, dysphagia, coronary heart disease, lower GCS score, chronic obstructive pulmonary disease, smoking, NIHSS scores, and glycemia ${ }^{15,16}$.

The stroke compromises voluntary and reflex cough, making the patient dysphagic and increasing the risk of aspiration due to the absence of airway protection mechanisms caused by the decrease or absence of laryngopharyngeal sensitivity, as well as damage to the motor function, compromising the oropharyngeal control ${ }^{17}$.

The male gender was identified as a risk factor for aspiration pneumonia. This data broadens the discussion about the health needs of men, not only biologically or epidemiologically, but also from a sociocultural perspective. It is known that men seek healthcare less frequently and adhere less to therapeutic behaviors ${ }^{18}$.

Factors related to advanced age and mechanical ventilation after 48 hours, as mentioned in the study, have been discussed in the literature and are widely variable, due to the demographic and clinical differences of the studied population. However, when ventilator-associated aspiration pneumonia (VAP) is correlated with elderly patients (mean age of 63.7 $\pm 11.5,71.5 \pm 12.7$ years old), it is an aggravating factor associated with multiple comorbidities, such as coronary diseases, increasing the mortality rate ${ }^{16}$. 
Table 4. Risk of individual bias specific to each type of study according to the Joanna Briggs Institute checklist ${ }^{11}$

\begin{tabular}{|c|c|c|c|c|c|c|c|c|c|c|c|c|}
\hline Authors and Year & Q1 & Q2 & Q3 & Q4 & Q5 & Q6 & Q7 & Q8 & & & \% Yes / Risk & Risk of Bias \\
\hline Masrur et al. & -- & $\sqrt{ }$ & -- & $\sqrt{ }$ & $\sqrt{ }$ & $\sqrt{ }$ & -- & $\sqrt{ }$ & & & 62.5 & $\begin{array}{c}++ \\
\text { Moderate }\end{array}$ \\
\hline Liu et al. & $\sqrt{ }$ & $\sqrt{ }$ & $\sqrt{ }$ & $\sqrt{ }$ & $\sqrt{ }$ & -- & $\sqrt{ }$ & $\sqrt{ }$ & & & 87.5 & $\begin{array}{c}+ \\
\text { Low }\end{array}$ \\
\hline Authors and Year & Q1 & Q2 & Q3 & Q4 & Q5 & Q6 & Q7 & Q8 & Q9 & Q10 & $\%$ Yes / Risk & Risk of Bias \\
\hline Ishigami et al. & $\sqrt{ }$ & $\sqrt{ }$ & $\sqrt{ }$ & I & $\sqrt{ }$ & $\sqrt{ }$ & $\sqrt{ }$ & $\sqrt{ }$ & $\sqrt{ }$ & $\sqrt{ }$ & 87.5 & $\begin{array}{c}+ \\
\text { Low }\end{array}$ \\
\hline
\end{tabular}

Cross Sectional Studies: Q1: Were the criteria for inclusion in the sample clearly defined?; Q2: Were the study subjects and the setting described in detail?; Q3: Was the exposure measured in a valid and reliable way?; Q4: Were objective, standard criteria used for measurement of the condition?; Q5: Were confounding factors identified?; Q6: Were strategies to deal with confounding factors stated?; Q7: Were the outcomes measured in a valid and reliable way?; Q8: Was appropriate statistical analysis used?. Case Control Studies:Q1: Were the groups comparable other than the presence of disease in cases or the absence of disease in controls?; Q2: Were cases and controls matched appropriately?; Q3: Were the same criteria used for identification of cases and controls?; Q4: Was exposure measured in a standard, valid and reliable way?; Q5: Was exposure measured in the same way for cases and controls?; Q6: Were confounding factors identified?; Q7: Were strategies to deal with confounding factors stated?; Q8: Were outcomes assessed in a standard, valid and reliable way for cases and controls?; Q9: Was the exposure period of interest long enough to be meaningful?; Q10: Was appropriate statistical analysis used?; V: Yes; --: No; I: Uncertain; NA: Not applicable; +++: High; ++: Moderate; +: Low

Another study related the old age to the fact that these patients had their physiological functions impaired ${ }^{19}$. Elderly people undergo anatomical and physiological changes related to the natural process of aging, impairing swallowing, increasing the risk of aspiration pneumonia ${ }^{19,20}$.

Therefore, neurological stability should be considered since the lower GCS score was significantly associated with aspiration pneumonia. The literature indicates that impaired consciousness is an independent factor for $\mathrm{AP}^{16}$. The study ${ }^{12}$ did not cite exactly the value of the GCS score to be considered as a reference parameter. However, the literature indicates that, for speech and language evaluation, patients should be attentive, maintaining sustained attention for more than fifteen minutes and a GCS $>14{ }^{21}$.

The aspiration of colonized gastric juice ${ }^{14}$ was one of the causing factors of aspiration pneumonia, corroborating with other researchers who reported that the aspiration of gastric contents was an important factor in determining the severity of pneumonitis, with the possibility of later development into aspiration pneumonia, respiratory failure or syndrome of acute respiratory distress ${ }^{22,23}$.

In the population of this research, no studies that indicated measures to prevent aspiration pneumonia were found. However, the literature highlights the damage covered by this demand, showing the need for higher quality researches.

A recent study in patients with total joint arthroplasty demonstrated the effectiveness of pulmonary screening together with the use of an intervention protocol developed to identify and prevent pulmonary complications. Risk screening identified all patients who presented pulmonary complications; only seven of the $7,658(0.091 \%)$ patients had pulmonary complications after the protocol started, which demonstrated its effectiveness in the prevention of pulmonary complications ${ }^{24}$. We considered the quality analysis of the evidence presented in the study as satisfactory, although needing improvements in some aspects. Mostly, the sample was significant for the evaluation, with objectives and methodologies well designed.

This systematic review addressed researches in hospitalized elderly patients, which limited the number of included articles. The extensive list of studies with populations of different ages and clinical profiles makes it difficult to analyze homogeneous samples. Therefore, there is a need for studies with well-defined samples, especially studies on the preventive measures of aspiration pneumonia in hospitalized elderly, to guide the implementation of strategies in care delivery and patient safety.

\section{CONCLUSION}

Severe stroke, dysphagia, old age, male gender, coronary heart disease, lower Glasgow coma scores, use of mechanical ventilation for more than 48 hours, and aspiration of colonized gastric juice can be considered factors for the development of pneumonia aspiration. On the other hand, a small number of studies in this review may limit our evidence.

No studies on the prevention of aspiration pneumonia in hospitalized elderly were found. These findings are of great importance for planning hospital 
speech therapy, as they will enable the development of specific aspiration pneumonia protocols for the elderly, contributing to more reliable health services.

\section{REFERENCES}

1. Nespollo AM, Marcon SR, Lima NVP, Dias TL, Martínez Espinosa M. Health Conditions and memory performance: a study with older adult women. Rev Bras Enferm. 2017;70(3):640-6.

2. Hollaar VRY, Putten GVD, Maarel-wierink CDVD, Bronkhorst EM, Swart BJD, Creugers NHJ. The effect of a daily application of a $0.05 \%$ chlorhexidine oral rinse solution on the incidence of aspiration pneumonia in nursing home residents: a multicenter study. BMC Geriatric. 2017;17(128):1-11.

3. Almeida AEM, Alcântara ACC, Lima FAM, Rocha HAL, Cremonin Junior JR, Costa HJM. Prevalência de risco moderado e alto de aspiração em pacientes hospitalizados e custo-efetividade da aplicação de protocolo preventivo. J Bras Econ Saúde. 2016; 8(3):216-20.

4. Osborne S, Gardner G, Gardner A, Franklin S, Tuohy E, Fisher A. Using a monitored sip test to assess risk of aspiration in postoperative patients. AORN J. 2006;83(4):908-28.

5. Dibardino DM, Wunderink RG. Aspiration pneumonia: a review of modern trends. $\mathrm{J}$ Crit Care. 2015;30(1):40-8.

6. Ramya K, Adhikari Prabha A, Sheetal R. Cost and antibiotic utilization of pneumonia patients in intensive care unit. J Appl Pharm Science. 2016;6(2):87-90.

7. Silva-Batalha EMS, Melleiro MM. Cultura de segurança do paciente em um hospital de ensino: diferenças de percepção existentes nos diferentes cenários dessa instituição. Enferm. 2015;24(2):432-41.

8. Palese A, Lesa L,Stroppolo G, Tardivo S, Brusaferro $S$. Factors precipitating the risk of aspiration in hospitalized patients: findings from a multicentre critical incident technique study. Int J Qual Health Care. 2017;29(2):194-9.

9. Lima IB, Pernambuco L. Hospital morbidity from stroke and speech-language therapy coverage in the state of Paraiba, Brazil. Audiol Commun Res. 2017;22 (e1822):1-7.

10. Moher D, Liberati A, Tetzlaff J, Altman DG, The PRISMA group. Preferred reporting items for systematic reviews and meta-analyses: the PRISMA statement. PLoS Med. 2009; 6(7):e1000097.
11. The Joanna Briggs Institute. Joanna Briggs Institute Reviewers' Manual: 2016 edition. Australia: The Joanna Briggs Institute; 2016.

12. Ishigami $K$, Okuro $M$, Koizumi $Y$, Satoh $K$, Iritani $O$, Yano $\mathrm{H}$ et al. Association of severe hypertension with pneumonia in elderly patients with acute ischemic strok. Hypertens Res. 2012;35(6):648-53.

13. Masrur S, Smith EE, Saver JL, Reeves MJ, Bhatt DL, Zhao JX et al. Dysphagia screening and hospitalacquired pneumonia in patients with acute ischemic stroke: findings from get with the guidelines-stroke. J Stroke Cerebrovasc Dis. 2013;22(8):e301-9.

14. Liu Q, Zhang I, Lin D, Mou X, He L, Qu J et al. Gastropulmonary route of infection and the prevalence of microaspiration in the elderly patients with ventilator associated pneumonia verified by molecular microbiology-GMPFGE. Cell Biochem Biophys. 2015;71(3):1457-62.

15. Ji R, Shen H, Yuesong P, Wang P, Liu G, Wang $Y$ et al. Novel risk score to predict pneumonia after acute ischemic stroke. Stroke. 2013;44(5):1303-9.

16. Othman HA, Gamil NM, Elgazzar AEM, Fouad TA. Ventilator associated pneumonia, incidence and risk factors in emergency intensive care unit Zagazig university hospitals. Egypt $\mathrm{J}$ Chest Dis Tuberc. 2017;66(4):703-8.

17. Kulnik ST, Birring SS, Hodsoll J, Moxham J, Rafferty GF, Kalra L. Higher cough flow is associated with lower risk of pneumonia in acute stroke. Thorax Month. 2016;71(5):474-5.

18. Araújo TU, Félix NDC, Ramos NM, Oliveira CJ, Almeida AIM. Nursing diagnosis Noncompliance to treatment in men with hypertension. Rev Rene. 2016;17(3):338-45.

19. Miki $Y$, Makuuchi R, Honda S, Tokunaga M, Tanizawa $\mathrm{Y}$, Bando $\mathrm{E}$ et al. Prospective phase II study evaluating the efficacy of swallow ability screening tests and pneumonia prevention using a team approach for elderly patients with gastric cancer. Gastric Cancer. 2018;21(2):353-9.

20. Silva TH, Massetti T, Silva TD, Paiva LS, Papa DCR, Monteiro CBM et al. Influence of severity of traumatic brain injury at hospital admission on clinical outcomes. Fisioter Pesqui. 2018;25(1):3-8.

21. Padovani AR, Moraes DP, Sassi FC, Andrade CRF. Clinical swallowing assessment in intensive care unit. CoDAS. 2013;25(1):1-7.

22. Wang EL, Lu J. Perioperative pulmonary aspiration prophylaxis. Essent Clinic Anesth Rev. 2015;6(39):143-6. 
23. Samantaray A. Pulmonary aspiration of gastric contents: prevention and prophylaxis. J Clin Sci Res. 2014;3(4):243-50.

24. Grau L, Orosco FR, Duque AF, Post MD, Ponzio DY, Ong AC. Simple protocol to stratify pulmonary risk reduces complications after total joint arthroplasty. J Arthroplasty. 2019;34(6):1233-9. 